\title{
Holographic entanglement entropy with Born-Infeld electrodynamics in higher dimensional AdS black hole spacetime
}

\author{
Weiping Yao ${ }^{1}$, Wenqing Zha ${ }^{1}$, Qiannan An ${ }^{1}$, Jiliang Jing ${ }^{2}$, a \\ ${ }^{1}$ Department of electrical engineering, Liupanshui Normal University, Liupanshui 553004, Guizhou, People's Republic of China \\ 2 Department of Physics, Key Laboratory of Low Dimensional Quantum Structures and Quantum Control of Ministry of Education, and Synergetic \\ Innovation Center for Quantum Effects and Applications, Hunan Normal University, Changsha 410081, Hunan, People's Republic of China
}

Received: 8 January 2019 / Accepted: 3 February 2019 / Published online: 19 February 2019

(C) The Author(s) 2019

\begin{abstract}
We examine the entanglement entropy in higher dimensional holographic metal/superconductor model with Born-Infeld (BI) electrodynamics. We note that the entanglement entropy is still a powerful tool to probe the critical phase transition point and the order of the phase transition in higher dimensional AdS spacetime. Due to the presence of the BI electromagnetic field, the formation of the scalar condensation becomes harder. For both operators $\left\langle\mathcal{O}_{+}\right\rangle$and $\left\langle\mathcal{O}_{-}\right\rangle$, we show that the entanglement entropy in the metal phase decreases as the BI factor increases, but in condensation phase the entanglement entropy increases monotonically for stronger nonlinearity of BI electromagnetic field. Furthermore, we also study the influence of the width of the subsystem on the holographic entanglement entropy and observe that with the increase of the width the entanglement entropy increases.
\end{abstract}

\section{Introduction}

The entanglement entropy serves as a key quantity to measure how the subsystem and its complement are correlated [1]. In strongly coupled system, the entanglement entropy is expected to be a useful tool to keep track of the degree of freedom while other traditional methods might not be available. However, the calculation of entanglement entropy is usually a not easy task except for the case in $1+1$ dimensions. In the spirit of the anti-de Sitter/conformal field theory (AdS/CFT) correspondence [2-4], Ryu and Takayanagi have provided a holographic proposal to compute the entanglement entropy in $[5,6]$. It states that the entanglement entropy of a d+1 dimensional CFT at strong coupling can be investigated from a weakly coupled gravity dual characterized by an asymptotically $\mathrm{AdS}_{d}+2$ spacetime. With this elegant approach, the

\footnotetext{
a e-mail: jljing@ @unnu.edu.cn
}

holographic entanglement entropy is widely used to study properties of phase transitions in holographic superconductor models [7-12]. The behavior of the entanglement entropy in metal/superconductor phase transition was studied in [13] and observed that the entanglement entropy is lower in the superconductor phase than in the normal phase. In the insulator/superconductor model, the non-monotonic behavior of the entanglement entropy was found in Ref. [14]. Then, the study of entanglement entropy was also extended to other various holographic superconductors applications [15-26].

The purpose of this paper is to study further the behaviors of the holographic entanglement entropy for metal/superconductor model with BI electrodynamics in higher dimensional AdS spacetime. On the one hand, the motivations for studying the entanglement entropy in higher dimensional black hole spacetime comes from the string theory which contains gravity and requires more than four dimensions $[27,28]$. Furthermore, as mathematical objects, black hole spacetimes are among the most important Lorentzian Ricciflat manifolds in any dimension [29]. On the other hand, the BI electrodynamics [30] introduced in 1930s by Born and Infeld to obtain a classical theory of charged particles with finite self-energy, is the only possible non-linear version of electrodynamics which is invariant under electromagnetic duality transformations and has been a focus for these years since most physical systems are inherently nonlinear to some extent [31-38]. The Lagrangian of the BI gauge field and its expression is given by

$L_{B I}=\frac{1}{b^{2}}\left(1-\sqrt{1+\frac{b^{2} F^{\mu \nu} F_{\mu \nu}}{2}}\right)$,

where $F_{\mu \nu}=\partial_{\mu} A_{\nu}-\partial_{\nu} A_{\mu}$ is the strength of the BI electrodynamic field. $b$ is the BI coupling parameter. In the limit $b \rightarrow 0$, the BI field will reduce to the Maxwell field. It is 
also to be noted that the higher order terms in the factor $b$ essentially correspond to the higher derivative corrections of the gauge fields $[39,40]$. In the present work, we will study the properties of phase transitions of the holographic superconductor model by calculating the behaviors of the scalar operator and the entanglement entropy and see how the phase transition is affected by the BI electrodynamics and the width of the subsystem in higher dimensional AdS black hole spacetime.

The framework of this paper is as follows. In Sect. 2, we introduce the basic field equations of holographic superconductor model with BI electrodynamics in n-dimensional spacetime and study the properties of the phase transition by calculating the scalar operator. In Sect. 3, the behavior of the entanglement entropy in the holographic superconductor model are investigated in derail. In Sect. 4, we conclude our main results of this paper.

\section{Holographic superconducting model with BI electrodynamics}

\subsection{Basic field equations}

In this section, we will consider the n-dimensional action in a background of AdS spacetime which includes Einstein gravity, a massive charged complex scalar field and BI nonlinear gauge field.

$$
\begin{aligned}
S= & \int d^{d} x \sqrt{-g}\left[\frac{1}{16 \pi G}(R-2 \Lambda)-\mid \nabla \psi\right. \\
& \left.-\left.i q A \psi\right|^{2}-m^{2}|\psi|^{2}+L_{B I}\right],
\end{aligned}
$$

where $R$ and $g$ are, respectively, the Ricci scalar curvature and the determinant of the metric, $\Lambda=-(d-1)(d-2) / 2 L^{2}$ is the cosmological constant, $L$ is the AdS radius, $\psi$ represents a scalar field with charge $q$ and mass $m$, and $L_{B I}$ is the Lagrangian of the BI gauge field.

In order to study the holographic entanglement entropy in BI electrodynamics, we will take the full backreaction. The metric ansatz for the d-dimensional planar black hole can be taken as

$d s^{2}=-f(r) e^{-\chi(r)} d t^{2}+\frac{d r^{2}}{f(r)}+r^{2} h_{i j} d x^{i} d x^{j}$,

where $h_{i j} d x^{i} d x^{j}$ is the line element of (d-2)-dimensional hypersurface with the curvature $\kappa=0$. The Hawking temperature of this black hole, which will be interpreted as the temperature of the CFT, reads

$T_{H}=\frac{f^{\prime}\left(r_{+}\right) e^{-\chi\left(r_{+}\right) / 2}}{4 \pi}$, where $r_{+}$is the black hole horizon. Assuming the matter fields in the forms

$A=\phi(r) d t, \quad \psi=\psi(r)$,

where $\psi(r)$ can be taken to be real without loss of generality. By varying action Eq. (2) with respect to the gravitational field $g_{\mu \nu}$, the scalar field $\psi$ and the gauge field $A_{\mu}$, the field equations can be obtained as

$$
\begin{aligned}
& \chi^{\prime}+\frac{2 r}{d-2}\left(\psi^{\prime 2}+\frac{q^{2} e^{\chi} \phi^{2} \psi^{2}}{f^{2}}\right)=0, \\
& f^{\prime}-\left(\frac{(d-1) r}{L}-\frac{(d-3) f}{r}\right) \\
& +\frac{r}{d-2}\left[m^{2} \psi^{2}+f\left(\psi^{\prime 2}+\frac{q^{2} e^{\chi} \phi^{2} \psi^{2}}{f^{2}}\right)\right. \\
& +\frac{1-\sqrt{1-b^{2} e^{\chi} \phi^{\prime 2}}}{\left.b^{2} \sqrt{1-b^{2} e^{\chi} \phi^{\prime 2}}\right]=0,} \\
& \psi^{\prime \prime}+\left(\frac{d-2}{r}-\frac{\chi^{\prime}}{2}+\frac{f^{\prime}}{f}\right) \psi^{\prime} \\
& +\frac{1}{f}\left(\frac{q^{2} e^{\chi} \phi^{2}}{f}-m^{2}\right) \psi=0, \\
& \phi^{\prime \prime}+\left(\frac{d-2}{r}+\frac{\chi^{\prime}}{2}\right) \phi^{\prime}-\frac{(d-2) b^{2} e^{\chi}}{r} \phi^{\prime 3} \\
& -\frac{2 q^{2} \psi^{2}\left(1-b^{2} e^{\chi} \phi^{\prime 2}\right)^{\frac{3}{2}}}{f} \phi=0,
\end{aligned}
$$

where a prime denotes the derivative with respect to $r$, and $16 \pi G=1$ was used. For purpose of getting the solutions in superconducting phase where $\psi(r) \neq 0$, we need to impose the boundary conditions. At the horizon $r_{+}$, the regularity condition gives the boundary conditions [41]

$$
\begin{aligned}
& \phi(r)=\phi_{1}\left(r-r_{+}\right)+\phi_{2}\left(r-r_{+}\right)^{2}+\cdots, \\
& \psi(r)=\psi_{0}+\psi_{1}\left(r-r_{+}\right)+\psi_{2}\left(r-r_{+}\right)^{2}+\cdots, \\
& \chi(r)=\chi_{0}+\chi_{1}\left(r-r_{+}\right)+\chi_{2}\left(r-r_{+}\right)^{2}+\cdots, \\
& f(r)=f_{1}\left(r-r_{+}\right)+f_{2}\left(r-r_{+}\right)^{2}+\cdots .
\end{aligned}
$$

And at the asymptotic AdS boundary $(r \rightarrow \infty)$, the asymptotic behaviors of the solutions are

$\chi \rightarrow 0, \quad f \sim \frac{r^{2}}{L^{2}} \quad \phi \sim \mu-\frac{\rho}{r^{d-3}} \quad \psi \sim \frac{\psi_{-}}{r^{\Delta_{-}}}+\frac{\psi_{+}}{r^{\Delta_{+}}}$

with

$\Delta_{ \pm}=\frac{1}{2}\left[(d-1) \pm \sqrt{(d-1)^{2}+4 m^{2}}\right]$

and $\mu$ and $\rho$ are interpreted as the chemical potential and charge density in the dual field theory. Considering Breitenlohner-Freedman bound [42,43], the mass of the scalar field must be restricted as $m^{2}>-(d-1)^{2} / 4$. It should be noted that provided $\Delta_{-}$is larger than the unitarity bound, both $\psi_{-}$and $\psi_{+}$can be normalizable [44]. This means $\psi_{-}$ 
or $\psi_{+}$can either be identified as a source or an expectation value. According to the AdS/CFT correspondence, they correspond to the vacuum expectation values $\psi_{-}=\left\langle\mathcal{O}_{-}\right\rangle$, $\psi_{+}=\left\langle\mathcal{O}_{+}\right\rangle$of an operator $\mathcal{O}$ dual to the scalar field $[45,46]$. Without loss of generality, we will impose boundary condition that either $\psi_{-}$or $\psi_{+}$vanishes in the following calculation.

From the equations of motion for the system, we can get the useful scaling symmetries in the forms

$$
\begin{aligned}
& r \rightarrow \alpha r, \quad(x, y, z, t) \rightarrow(x, y, z, t) / \alpha, \\
& \phi \rightarrow \alpha \phi, \quad f \rightarrow \alpha^{2} f, \\
& L \rightarrow \alpha L, \quad r \rightarrow \alpha r, \quad t \rightarrow \alpha t, \quad q \rightarrow \alpha^{-1} q, \\
& e^{\chi} \rightarrow \alpha^{2} e^{\chi}, \quad \phi \rightarrow \alpha^{-1} \phi, \quad t \rightarrow t \alpha .
\end{aligned}
$$

Using the scaling symmetries Eq. (16), we can take $r_{+}=1$ and the symmetries Eq. (17) allow us to set the $L=1$. By applying the scaling symmetries Eq. (16), the useful quantities can be rescaled as

$$
\begin{aligned}
T & \rightarrow \alpha T, \quad \rho \rightarrow \alpha^{d-2} \rho, \quad\left\langle\mathcal{O}_{-}\right\rangle \rightarrow \alpha^{\Delta_{-}}\left\langle\mathcal{O}_{-}\right\rangle, \\
\left\langle\mathcal{O}_{+}\right\rangle & \rightarrow \alpha^{\Delta_{+}}\left\langle\mathcal{O}_{+}\right\rangle .
\end{aligned}
$$

Therefore, we will use the following dimensionless quantities in next section

$$
\left\langle\mathcal{O}_{+}\right\rangle^{\frac{1}{\Delta_{+}}} / \rho^{\frac{1}{d-2}}, \quad\left\langle\mathcal{O}_{-}\right\rangle^{\frac{1}{\Delta_{-}}} / \rho^{\frac{1}{d-2}}, \quad T / \rho^{\frac{1}{d-2}} .
$$

\subsection{Metal/superconductor phase transition}

After the discussion in the above section, we here would like to study the properties of phase transition of this physic model through the condensation of the scalar operators $\left\langle\mathcal{O}_{+}\right\rangle$and $\left\langle\mathcal{O}_{-}\right\rangle$. For concreteness, we investigate the behaviors of the scalar condensation for the system in five-dimensional AdS black hole space-time. Considering Breitenlohner-Freedman bound $[42,43]$, we here set $q=2$ and $m^{2}=\sqrt{-15 / 4}$. The behavior of the scalar condensation versus the temperature $T$ for different BI parameter $b$ is shown in Fig. 1. For a given $\mathrm{b}$, the left panel shows that as the temperature $T$ exceeds a critical value $T_{c}$, the scalar field is vanishing and this can be identified as the metal phase. However, the condensation of the operators emerges as $T<T_{c}$, which can be thought of as a superconductor phase. Near the phase transition point $T_{c}$, the critical behavior is found to be $\left\langle\mathcal{O}_{+}\right\rangle \propto\left(T_{c}-T\right)^{1 / 2}$, which means the phase transition is second order [45-48]. And it is noted that the curves for the scalar operators has similar behaviors to the BCS theory for different BI factor, where the condensation goes to a constant at zero temperature. In the right panel, it can be seen that the condensation of the operator $\left\langle\mathcal{O}_{-}\right\rangle$with respect of the temperature $\mathrm{T}$ is similar to the case of the operator $\left\langle\mathcal{O}_{+}\right\rangle$. As the temperature is fixed, we observe that with the increase of the BI parameter the condensation gaps increases for both scalar operators $\left\langle\mathcal{O}_{+}\right\rangle$ and $\left\langle\mathcal{O}_{-}\right\rangle$, which implies that the scalar hair can be formed harder when the nonlinearity in the BI electromagnetic field becomes stronger. Interestingly, the condensation gap of the operator $\left\langle\mathcal{O}_{+}\right\rangle$is larger than the one of the operator $\left\langle\mathcal{O}_{-}\right\rangle$as the $\mathrm{BI}$ factor changes in same value. That is to say, the effect of the $\mathrm{BI}$ factor on the condensation of operator $\left\langle\mathcal{O}_{+}\right\rangle$is more powerful than the one of operator $\left\langle\mathcal{O}_{-}\right\rangle$. From Table 1, we find that the critical temperature $T_{c}$ for the operators $\left\langle\mathcal{O}_{+}\right\rangle$ and $\left\langle\mathcal{O}_{-}\right\rangle$decreases as the $\mathrm{BI}$ factor increases, which agrees well with the results obtained in Fig. 1.

\section{Entanglement entropy in holographic phase transition}

In this section, we will study the phase transition in the holographic superconductor model with BI electrodynamics by the entanglement entropy. The entanglement entropy in con-
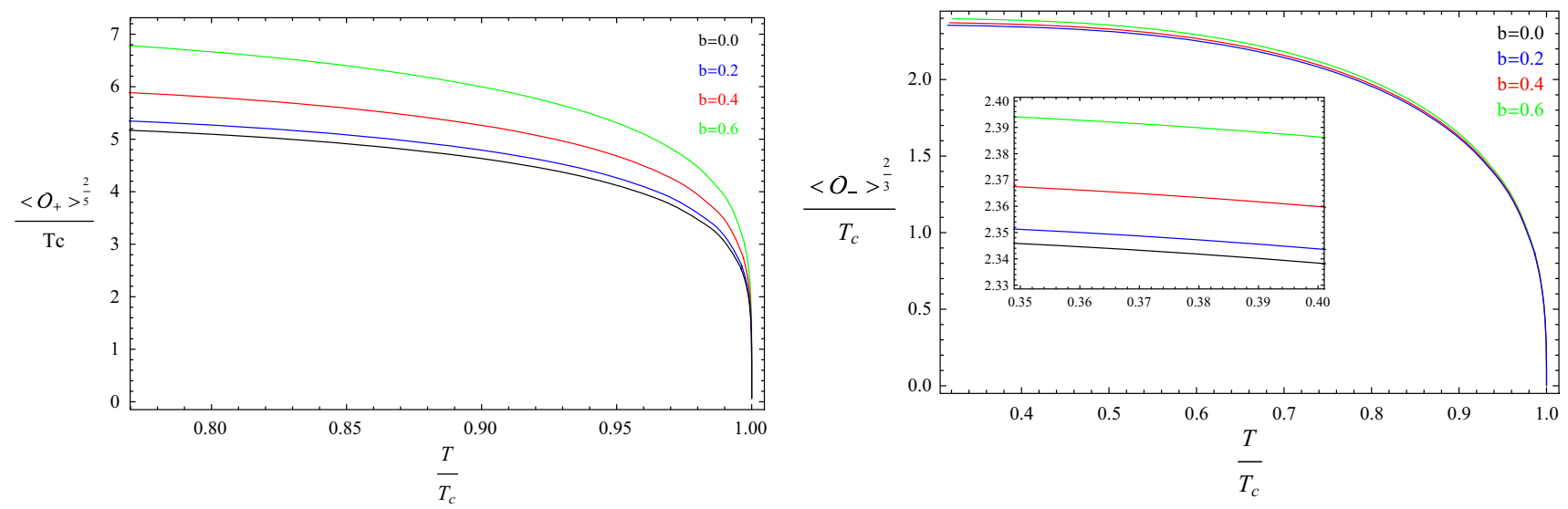

Fig. 1 The operators $\left\langle\mathcal{O}_{+}\right\rangle$(left plot) and $\left\langle\mathcal{O}_{-}\right\rangle$(right plot) with respect to the temperature $T$ after condensation for different BI parameter. The four lines from top to bottom correspond to $b=0$ (black), $b=0.2$ (blue), $b=0.4$ (red), and $b=0.6$ (green) respectively 
Table 1 The critical temperature $T_{c}$ for the operators $\left\langle\mathcal{O}_{+}\right\rangle$and $\left\langle\mathcal{O}_{-}\right\rangle$for different BI parameter

\begin{tabular}{llllllllll}
\hline$b$ & 0.0 & 0.1 & 0.2 & 0.3 & 0.4 & 0.5 & 0.6 & 0.7 & 0.8 \\
\hline$\left\langle\mathcal{O}_{+}\right\rangle$ & 0.1900 & 0.1884 & 0.1837 & 0.1761 & 0.1662 & 0.1542 & 0.1410 & 0.1269 & 0.1127 \\
$\left\langle\mathcal{O}_{-}\right\rangle$ & 0.3782 & 0.3780 & 0.3774 & 0.3763 & 0.3749 & 0.3730 & 0.3708 & 0.3683 & 0.3654 \\
\hline
\end{tabular}

formal field theories can be calculated from the area of minimal surface in AdS spaces, and its formula is given by the "area law" [5,6]

$S_{\mathcal{A}}=\frac{\operatorname{Area}\left(\gamma_{A}\right)}{4 G_{A}}$

where $\gamma_{A}$ is the minimal area surface in the bulk which ends on the boundary A, $G_{N}$ is the Newton constant in the Einstein gravity on the AdS space, and $S_{A}$ is the entanglement entropy for the subsystem A which can be chosen arbitrarily. Hereafter, we focus on the entanglement entropy for a straight geometry with a finite width $\ell$ along the $x$ direction and infinitely extending in $y$ and $z$ directions. The holographic dual surface $\gamma_{A}$ is defined as a three-dimensional surface

$$
\begin{aligned}
& t=0, \quad x=x(r), \quad-\frac{R}{2}<y<\frac{R}{2}(R \rightarrow \infty), \\
& -\frac{W}{2}<z<\frac{W}{2}(W \rightarrow \infty),
\end{aligned}
$$

and the holographic surface $\gamma_{A}$ in direction stats from $x=\frac{\ell}{2}$ at $r=\frac{1}{\epsilon}$, extends into the bulk until it reaches $r=r_{*}$, then returns back to the AdS boundary $r=\frac{1}{\epsilon}$ at $x=-\frac{\ell}{2}$. The induced metric on the hypersurface $A$ is

$d s^{2}=h_{i j} d x^{i} d x^{j}$

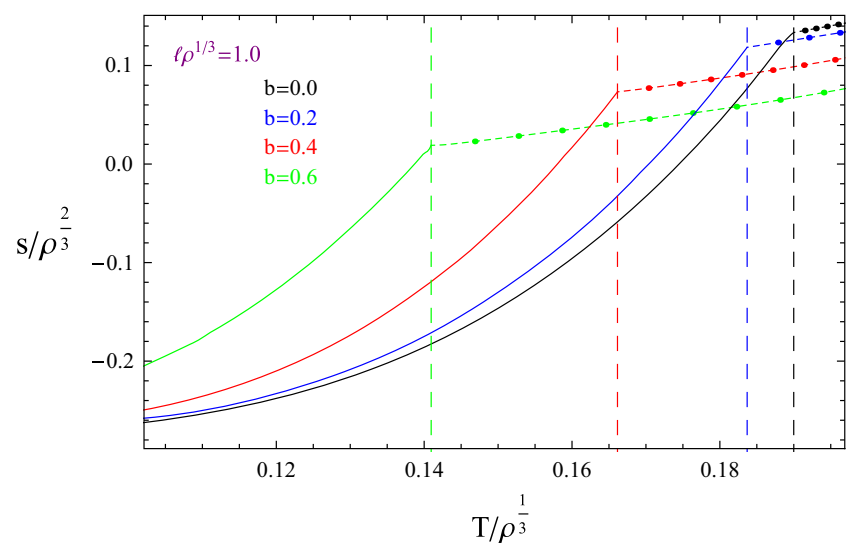

$$
=\left(\frac{1}{f(r)}+r^{2}\left(\frac{d x}{d r}\right)^{2}\right) d r^{2}+r^{2} d y^{2}+r^{2} d z^{2} \text {. }
$$

According to the Eq. (21), the entanglement entropy in the strip geometry is

$S_{\mathcal{A}}[x]=\frac{R W}{2 G_{5}} \int_{r_{*}}^{\frac{1}{\epsilon}} r^{2} \sqrt{\frac{1}{f(r)}+r^{2}(d x / d r)^{2}} d r$

where $r=\frac{1}{\epsilon}$ is the UV cutoff. Noting that the above expression can be treated as the Lagrangian with $\mathrm{x}$ direction thought of as time. Therefore, the equation of motion for the minimal surface from Eq. (24) is given by

$\frac{r^{4}(d x / d r) \sqrt{f(r)}}{\sqrt{1+r^{2} f(r)(d x / d r)^{2}}}=r_{*}^{3}$.

We are interested in the case that the surface is smooth at $r=r_{*}$ i.e. $d x /\left.d r\right|_{r=r_{*}}=0$. By using the variable $z_{s}=1 / r$ , the width $\ell$ in terms of $z_{s}$ is

$\frac{l}{2}=\int_{\varepsilon}^{z_{*}} d z_{s} \frac{z_{s}^{3}}{\sqrt{\left(z_{*}^{6}-z_{s}^{6}\right) z_{s}^{2} f\left(z_{s}\right)}}$

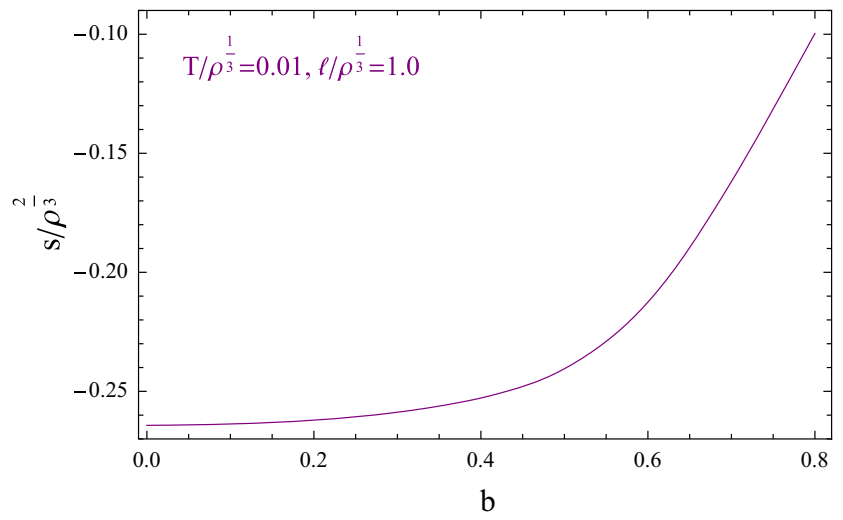

Fig. 2 The entanglement entropy of the operator $\left\langle O_{+}\right\rangle$with respect to the temperature $T$ and $\mathrm{BI}$ factor $\mathrm{b}$ for $\ell \rho^{\frac{1}{3}}=1$. The four lines in left plot from top to bottom correspond to $b=0$ (black), $b=0.2$ (blue), $b=0.4$ (red), and $b=0.6$ (green) respectively 


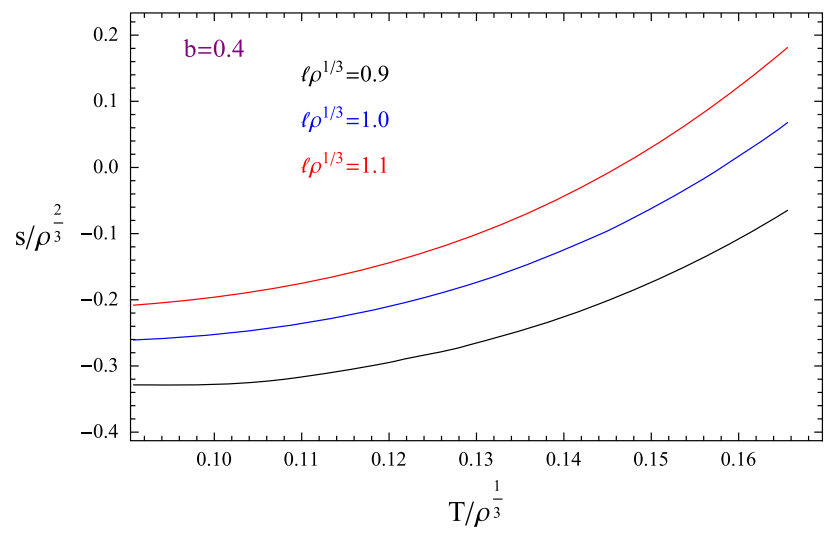

Fig. 3 The entanglement entropy of the operator $\left\langle O_{+}\right\rangle$with respect to the temperature $T$ for various widths $\ell$ as $b=0.4$. The red line is for $\ell \sqrt{\rho}=1.1$, the blue line is for $\ell \sqrt{\rho}=1.0$, and black one is for $\ell \sqrt{\rho}=0.9$

and the holographic entanglement entropy in the $z_{s}$-coordinate can be rewritten as

$$
\begin{aligned}
S_{\mathcal{A}} & =\frac{R W}{2 G_{5}} \int_{\varepsilon}^{z_{*}} d z_{s} \frac{z_{*}^{3}}{z_{s}^{2}} \frac{1}{\sqrt{\left(z_{*}^{6}-z_{s}^{6}\right) z_{s}^{2} f\left(z_{s}\right)}} \\
& =\frac{R W}{4 G_{5}}\left(\frac{1}{\varepsilon^{2}}+s\right),
\end{aligned}
$$

the first term $1 / \varepsilon$ is divergent as $\varepsilon \rightarrow 0$. However, the second term dose not depend on the cutoff and is finite, so it is physical important. In the following we will study the entanglement entropy in the holographic superconductor model and explore its dependence on the factors of the temperature $T$, the BI factor $b$ and the belt width $\ell$.

\subsection{Entanglement entropy for operator $\left\langle\mathcal{O}_{+}\right\rangle$}

We show the behavior of the entanglement entropy of operator $\left\langle\mathcal{O}_{+}\right\rangle$with respects to the temperature $T$ and $\mathrm{BI}$ factor $\mathrm{b}$ in Fig. 2 with the dimensionless quantities $s / \rho^{\frac{1}{3}}, \ell \rho^{\frac{1}{3}}, T / \rho^{\frac{1}{3}}$. The left panel shows the cases of $\ell \rho^{\frac{1}{3}}=1$ with various $T$ and the right panel is the cases of $T / \rho^{\frac{1}{3}}=0.1, \ell \rho^{\frac{1}{3}}=1$ with various $b$. It can be seen from the left-hand diagram that the slop of the entanglement entropy at the phase transition points indicated by the vertical dotted lines is discontinuous but the value of the entanglement entropy is continuous. Which indicates some kind of new degrees of freedom like Cooper pair would emerge after the condensation and this phase transition can be regarded as the signature of the second order phase transition. With the increase of the BI factor $b$ the critical temperature $T_{c}$ of the phase transition decreases which means that the stronger BI electrodynamics correction makes the scalar hair harder to condense. Moreover, the entanglement entropy in the superconductor phases for different $b$ denoted by the solid lines are lower than the ones in the normal phases represented by the dot-dashed lines and decreases monotonously as temperature decreases. That is to say, the scalar hair turns on at the critical temperature and the formation of Cooper pairs make the degrees of freedom decrease in the superconductor phase. As the factor $b$ becomes lager entanglement entropy in the mental phase decreases. In the condensation phase, it can be seen from the right diagram that the entanglement entropy increases monotonically for bigger $b$ and this behavior of entanglement entropy is quite different from the results discussed in four-dimensional spacetime [49], where the entanglement entropy first increases and forms a peak at the threshold then decreases monotonically with increase of the BI parameter.
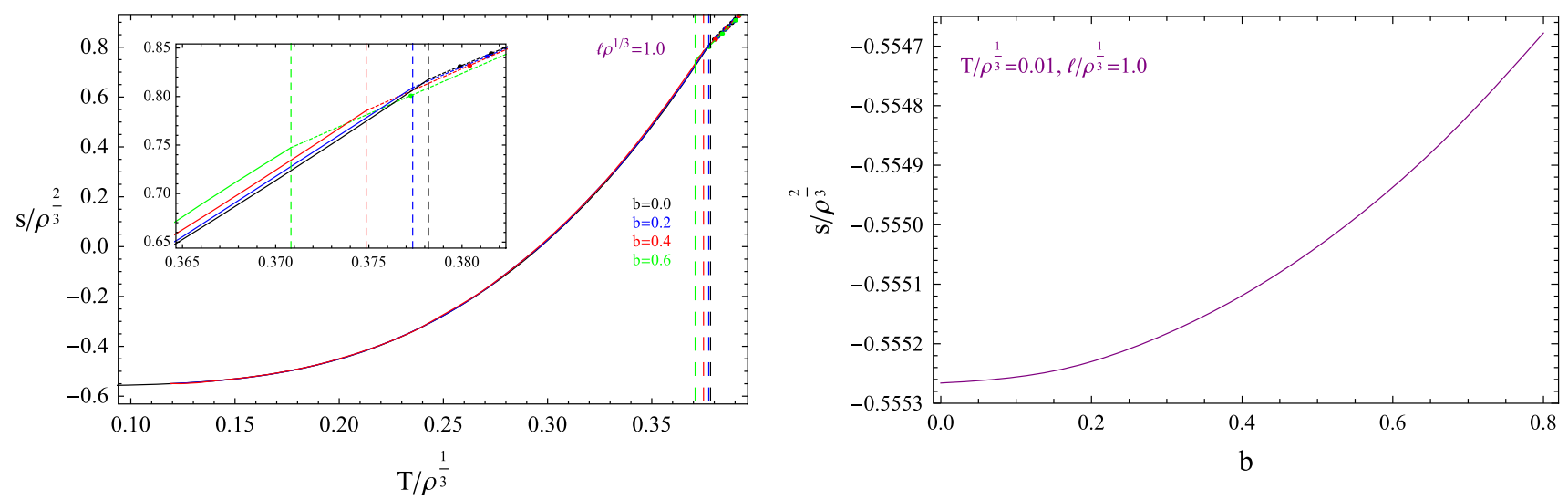

Fig. 4 The entanglement entropy of the operator $\left\langle O_{-}\right\rangle$with respect to the temperature $T$ and $\mathrm{BI}$ factor $\mathrm{b}$ for $\ell \rho^{\frac{1}{3}}=1$. The four lines in left plot from top to bottom correspond to $b=0$ (black), $b=0.2$ (blue), $b=0.4$ (red), and $b=0.6$ (green) respectively 


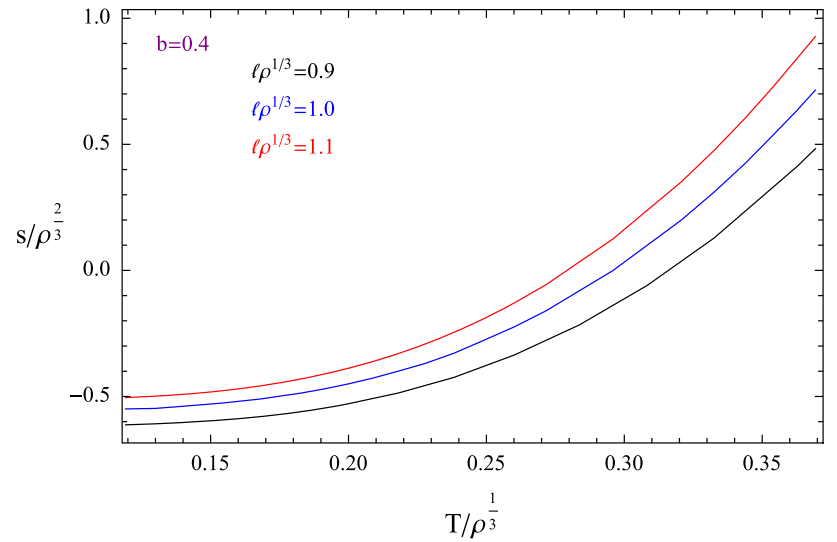

Fig. 5 The entanglement entropy of the operator $\left\langle O_{-}\right\rangle$with respect to the temperature $T$ for various widths $\ell$ as $b=0.4$. The red line is for $\ell \sqrt{\rho}=1.1$, the blue line is for $\ell \sqrt{\rho}=1.0$, and black one is for $\ell \sqrt{\rho}=0.9$

To get further understanding of the influence of the width $\ell$ on the entanglement entropy in the superconductor phase, we plot the corresponding results in Fig. 3. For a given $b$, we find that the entanglement entropy becomes smaller as the temperature $T$ gets lower. With the growth of the belt width $\ell$ the value of the entanglement entropy $s$ increases.

\subsection{Entanglement entropy for operator $\left\langle\mathcal{O}_{-}\right\rangle$}

The dependence of the entanglement entropy for operator $\left\langle\mathcal{O}_{-}\right\rangle$on the temperature and the $\mathrm{BI}$ factor is exhibited in Fig. 4. The vertical dotted lines represent the critical temperature of the phase transition for the different value of the BI factor. The dashed line is from the metal phase and the solid one is from the superconductor phase. In the left panel, the critical temperature of phase transition decreases as the BI parameter becomes bigger. Which indicates the scalar hair becomes harder to be formed as the nonlinearity in the electromagnetic field increases. We also find that there exists a jump of the slop of the entanglement entropy at the phase transition point . In the right panel, the behavior of the entanglement entropy of operator $\left\langle\mathcal{O}_{-}\right\rangle$as a function of the BI factor is similar to the case of the operator $\left\langle O_{+}\right\rangle$. However , the effect of the BI factor on the entanglement entropy of operator $\left\langle\mathcal{O}_{-}\right\rangle$is weaker than the one of operator $\left\langle\mathcal{O}_{+}\right\rangle$. In Fig. 5, it is shown that the influence of the width $\ell$ on the entanglement entropy is analogous to that of Fig. 3 where the entanglement entropy increases as the width $\ell$ increases.

\section{Conclusion}

In this paper, we calculated the holographic metal/ superconductor phase transition with BI electrodynamics in higher dimensional AdS spacetime. We first explored the properties of the phase transition of this system by analyzing the behaviors of the scalar operator and found that the value of the critical temperature $T_{c}$ of the phase transition becomes smaller as the $\mathrm{BI}$ factor $b$ increases. This means that the BI correction to the usual Maxwell field hinders the formation of the scalar hair. By comparing the effects of the BI parameter on the condensation, we observer that the effect of the $\mathrm{BI}$ factor on the condensation of operator $\left\langle\mathcal{O}_{+}\right\rangle$is more powerful than the one of operator $\left\langle\mathcal{O}_{-}\right\rangle$. Instead of the probe limit, when taking the full-backreaction into consideration, we observed that the curves for two scalar operators has similar behaviors to the BCS theory for different BI factor. In addition, to further study the properties of the phase transition, we used holographic methods to explored the behavior of the entanglement entropy in the holographic model. For both operators $\left\langle\mathcal{O}_{+}\right\rangle$and $\left\langle\mathcal{O}_{-}\right\rangle$, the critical temperature $T_{c}$ of the phase transition obtained from the behaviors of entanglement entropy is the same as the threshold temperature obtained from the behaviors of the scalar operator. And the discontinuous slop of the entanglement entropy at the critical temperature $T_{c}$ corresponds to second order phase transition in this physical system. Consequently, the entanglement entropy is indeed a good probe to study the properties of the phase transition, and that its behavior can indicate not only the appearance, but also the order of the phase transition. Furthermore, the entanglement entropy of operator $\left\langle\mathcal{O}_{+}\right\rangle$in the condensation phase increases monotonically for bigger $b$ and this behavior of entanglement entropy is quite different from the results discussed in four-dimensional spacetime [49], in which the result of the entanglement entropy first increases and forms a peak at the threshold then decreases monotonically with increase of the BI parameter.

Acknowledgements This work was supported by the National Natural Science Foundation of China under Grant nos. 11665015, 11875025; Guizhou Provincial Science and Technology Planning Project of China under Grant no. qiankehejichu[2016]1134; The talent recruitment program of Liupanshui normal university of China under Grant no. LPSSYKYJJ201508.

Data Availability Statement This manuscript has no associated data or the data will not be deposited. [Authors' comment: This manuscript concerns only to theoretical investigations and therefore does not contain any associated data.]

Open Access This article is distributed under the terms of the Creative Commons Attribution 4.0 International License (http://creativecomm ons.org/licenses/by/4.0/), which permits unrestricted use, distribution, and reproduction in any medium, provided you give appropriate credit to the original author(s) and the source, provide a link to the Creative Commons license, and indicate if changes were made.

Funded by SCOAP ${ }^{3}$.

\section{References}

1. S. Ryu, Y. Hatsugai, Entanglement entropy and the Berry phase in the solid state. Phys. Rev. B 73, 245115 (2006). arXiv:cond-mat/0601237 
2. J.M. Maldacena, The large $\mathrm{N}$ limit of superconformal field theories andsupergravity Adv. Theor. Math. Phys. 2, 231-252 (1998). arXiv:hep-th/9711200

3. S.S. Gubser, I.R. Klebanov, Gauge theory correlators from noncritical string theory. A. M. Polyakov Phys. Lett. B 428, 105-114 (1998). arXiv:hep-th/9802109

4. E. Witten, Anti-de Sitter space and holography. Adv. Theor. Math. Phys. 2, 253-291 (1998). arXiv:hep-th/9802150

5. S. Ryu, T. Takayanagi, Holographic derivation of entanglement entropy from AdS/CFT. Phys. Rev. Lett. 96, 181602 (2006)

6. S. Ryu, T. Takayanagi, Aspects of holographic entanglement entropy. JHEP 0608, 045 (2006)

7. D.V. Fursaev, Proof of the holographic formula for entanglement entropy. JHEP 0609, 018 (2006)

8. T. Hirata, T. Takayanagi, AdS/CFT and strong subadditivity of entanglement entropy. JHEP 0702, 042 (2007)

9. T. Nishioka, T. Takayanagi, AdS bubbles, entropy and closed string tachyons. JHEP 0701, 090 (2007)

10. I.R. Klebanov, D. Kutasov, A. Murugan, Entanglement as a probe of confinement. Nucl. Phys. B 796, 274 (2008)

11. R.C. Myers, A. Singh, Comments on holographic entanglement entropy and RG flows. JHEP 1204, 122 (2012)

12. A. Pakman, A. Parnachev, Topological entanglement entropy and holography. JHEP 0807, 097 (2008)

13. T. Albash, C.V. Johnson, Holographic studies of entanglement entropy in superconductors. JHEP 05, 079 (2012)

14. R.-G. Cai, S. He, L. Li, Y.-L. Zhang, Holographic entanglement entropy in insulator/superconductor transition. JHEP 1207, 088 (2012)

15. R.-G. Cai, S. He, L. Li, L.-F. Li, Entanglement entropy and Wilson loop in Stckelberg holographic insulator/superconductor model. JHEP 1210, 107 (2012)

16. R.-G. Cai, L. Li, L.-F. Li, S. Ru-Keng, Entanglement entropy in holographic P-wave superconductor/insulator model. JHEP 1306, 063 (2013)

17. J. de Boer, M. Kulaxizi, A. Parnachev, Holographic entanglement entropy in Lovelock gravities. JHEP 1107, 109 (2011)

18. L.-Y. Hung, R.C. Myers, M. Smolkin, On holographic entanglement entropy and higher curvature gravity. JHEP 1104, 025 (2011)

19. N. Ogawa, T. Takayanagi, Higher derivative corrections to holographic entanglement entropy for AdS solitons. JHEP 1110, 147 (2011)

20. W. Yao, J. Jing, Holographic entanglement entropy in insulator/superconductor transition with Born-Infeld electrodynamics. JHEP 05, 058 (2014)

21. X. Dong, Holographic entanglement entropy for general higher derivative gravity. JHEP 01, 044 (2014)

22. X.-M. Kuang, E. Papantonopoulos, B. Wang, Entanglement entropy as a probe of the proximity effect in holographic superconductors. J. High Energy Phys. 1405, 130 (2014)

23. Y. Peng, Holographic entanglement entropy in superconductor phase transition with dark matter sector. Phys. Lett. B 750, 420-426 (2015)

24. X.-X. Zeng, H. Zhang, L.-F. Li, Phase transition of holographic entanglement entropy in massive gravity. Phys. Lett. B 756, 170 (2016)

25. N.S. Mazhari, D. Momeni, R. Myrzakulov, H. Gholizade, M. Raza, Non-equilibrium phase and entanglement entropy in 2D holographic superconductors via Gauge-String duality. Can. J. Phys. 10, 94 (2016)
26. Y. Peng, G. Liu, Holographic entanglement entropy in two-order insulator/superconductor transitions. Phys. Lett. B 767, 330-335 (2017)

27. A. Strominger, C. Vafa, Microscopic origin of the BekensteinHawking entropy. Phys. Lett. B 379, 99 (1996)

28. O. Aharony, S.S. Gubser, J.M. Maldacena, H. Ooguri, Y. Oz, Large $\mathrm{N}$ field theories, large $\mathrm{N}$ field theories, string theory and gravity. Phys. Rep. 323, 183 (2000)

29. R. Emparan, H.S. Reall, Black holes in higher dimensions. Living Rev. Relativ. 11, 6 (2008)

30. M. Born, L. Infeld, Foundations of the new field theory. Proc. R. Soc. A 144, 425 (1934)

31. G.W. Gibbons, D.A. Rasheed, Electric-magnetic duality rotations in non-linear electrodynamics. Nucl. Phys. 454, 185 (1995)

32. B. Hoffmann, Gravitational and electromagnetic mass in the BornInfeld electrodynamics. Phys. Rev. 47, 877 (1935)

33. W. Heisenberg, H. Euler, Folgerungen aus der Diracschen Theorie des Positrons. Z. Phys. 98, 714 (1936)

34. H.P. de Oliveira, Non-linear charged black holes. Class. Quant. Grav. 11, 1469 (1994)

35. J.L. Jing, S.B. Chen, Holographic superconductors in the BornInfeld electrodynamics. Phys. Lett. B 686, 68 (2010)

36. O. Miskovic, R. Olea, Conserved charges for black holes in Einstein-Gauss-Bonnet gravity coupled to nonlinear electrodynamics in AdS space. Phys. Rev. D 83, 024011 (2011)

37. Y. Q. Liu, Y. Peng, B. Wang, Gauss-Bonnet holographic superconductors in Born-Infeld electrodynamics with backreactions. arXiv: 1202.3586

38. W. Yao, J. Jing, Analytical study on holographic superconductors for Born-Infeld electrodynamics in Gauss-Bonnet gravity with backreactions. JHEP 05, 101 (2013)

39. D. Roychowdhury, Effect of external magnetic field on holographic superconductors in presence of nonlinear corrections. Phys. Rev. D 86, 106009 (2012)

40. D. Roychowdhury, AdS/CFT superconductors with Power Maxwell electrodynamics: reminiscent of the Meissner effect. Phys. Lett. B 718, 1089 (2013)

41. Y. Peng, Q. Pan, B. Wang, Various types of phase transitions in the AdS soliton background. Phys. Lett. B 699, 383-387 (2011)

42. P. Breitenlohner, D.Z. Freedman, Stability In gauged extended supergravity. Ann. Phys. 144, 249 (1982)

43. P. Breitenlohner, D.Z. Freedman, Positive energy in anti-De Sitter backgrounds and gauged extended supergravity. Phys. Lett. B 115, 197 (1982)

44. G.T. Horowitz, M.M. Roberts, Holographic superconductors with various condensates. Phys. Rev. D 78, 126008 (2008)

45. S.A. Hartnoll, C.P. Herzog, G.T. Horowitz, Building a holographic superconductor. Phys. Rev. Lett. 101, 031601 (2008)

46. S.A. Hartnoll, C.P. Herzog, G.T. Horowitz, Holographic superconductors. J. High Energy Phys. 12, 015 (2008)

47. Q. Pan, B. Wang, E. Papantonopoulos, J. de Oliveira, A.B. Pavan, Holographic superconductors with various condensates in Einstein-Gauss-Bonnet gravity. Phys. Rev. D 81, 106007 (2010)

48. Y. Peng, Y. Liu, A general holographic metal/superconductor phase transition mode. JHEP 02, 082 (2015)

49. W. Yao, J. Jing, Holographic entanglement entropy in metal/superconductor phase transition with Born-Infeld electrodynamics. Nucl. Phys. B 889, 109 (2014) 